\title{
Practical techniques for the vibration method with additional mass: effect of specimen moisture content
}

\author{
Yoshitaka Kubojima $^{1} \cdot$ Satomi Sonoda $^{2} \cdot$ Hideo Kato $^{1}$
}

Received: 2 May 2017/Accepted: 1 July 2017/Published online: 18 August 2017

(C) The Japan Wood Research Society 2017

\begin{abstract}
This work examines the effect of moisture content on the accuracy of nondestructively and simply estimating weight, density, and Young's modulus by a vibration test without measuring specimen weight. The resonance frequencies with and without concentrated mass were measured by longitudinal vibration and bending vibration tests. The wet specimens whose initial moisture contents were $93-134 \%$ were dried at $20^{\circ} \mathrm{C}$ and $65 \%$ relative humidity, and their weight, density, and Young's modulus were estimated. The accuracy of the estimation was affected by the increase in the resonance frequency, caused by the drying process, during the vibration tests. The resonance frequency without the concentrated mass should, therefore, be measured after obtaining the resonance frequency with the concentrated mass. The accuracy of the estimation in the bending vibration test was higher than that in the longitudinal vibration test. This tendency can be explained by the error in the measurement of resonance frequencies with and without the concentrated mass.
\end{abstract}

Keywords Bending vibration - Longitudinal vibration . Moisture content . Vibration method with additional mass . Wood

This study was presented in part at the 67th Annual Meeting of Japan Wood Research Society, Fukuoka, March 2017.

Yoshitaka Kubojima

kubojima@ffpri.affrc.go.jp

1 Forestry and Forest Products Research Institute, 1 Matsunosato, Tsukuba, Ibaraki 305-8687, Japan

2 Toyama Prefectural Agricultural, Forestry and Fisheries Research Center, 4940 Kurokawa Shin, Imizu, Toyama 939-0311, Japan

\section{Introduction}

A vibration test is a simple and nondestructive method to measure Young's modulus and can be applied to machine stress grading. Measurement of specimen density (weight) is required for this testing method; however, the weighing of each piled lumber and the removal of each beam from its timber guardrail for weighing are time-consuming and labor-intensive. Hence, a simple testing method to determine Young's modulus of lumber without measuring weight is required.

Using theories of vibration, the effect of an additional mass bonded to a bar on the Young's modulus of the bar has been previously investigated, and frequency equations incorporating the effect of the additional mass and its position for longitudinal and bending vibrations have been developed. These frequency equations can be used to obtain the density and Young's modulus values from vibration tests without weighing the specimen [1-13]. In the present study, this procedure is referred to as the "vibration method with additional mass".

Various test conditions must be assessed before applying this method to actual cases. The position of concentrated mass [14] and the effect of the crosser's position of piled lumber [15] have already been studied. The moisture content of piled lumber varies, because it is difficult to perform perfect wood drying. The moisture content of beams for timber guardrails varies because of rain. Thus, the question of whether the vibration method with additional mass can be applied to wet wood needs to be clarified. The effect of the wood moisture content on the vibration method with additional mass was investigated in this study. 


\section{Vibration method with additional mass}

In the case of a slender beam, the effect of shear deflection and rotary inertia involved in the flexural vibrational deflection is negligible, and the Euler-Bernoulli elementary theory of bending can be applied to the vibration.

Young's moduli using the longitudinal and bending vibrations $E$ of a rectangular bar with length $l$ are expressed as follows:

$E=\rho\left(\frac{l \omega_{\mathrm{n}}}{m_{\mathrm{n}}}\right)^{2}$ (Longitudinal vibration),

$E=\left(\frac{l}{m_{\mathrm{n}}}\right)^{4} \frac{\rho A}{I} \omega_{\mathrm{n}}^{2}$ (Bending vibration),

where $\rho, \omega, A$, and $I$ are the density, angular frequency $(\omega=2 \pi f, f:$ resonance frequency), cross-sectional area, and the moment of inertia of the cross section, respectively. The value of $m_{\mathrm{n}}$ is explained below.

The frequency equation for the free-free longitudinal and bending vibrations with concentrated mass $M$ placed at $x=a l$ ( $x$ : distance along the bar, $0 \leq a \leq 1, a+b=1$ ) of a rectangular bar (Fig. 1) is expressed as follows [7, 9]:

$\sin m_{\mathrm{n}}+\mu m_{\mathrm{n}} \cos a m_{\mathrm{n}} \cos b m_{\mathrm{n}}=0$ (Longitudinal),

$\left(\cos m_{\mathrm{n}} \cosh m_{\mathrm{n}}-1\right)-\frac{1}{2} \mu m_{\mathrm{n}}\left\{\left(\cos a m_{\mathrm{n}} \cosh a m_{\mathrm{n}}+1\right)\right.$

$\left(\sin b m_{\mathrm{n}} \cosh b m_{\mathrm{n}}-\cos b m_{\mathrm{n}} \sinh b m_{\mathrm{n}}\right)$

$\left.+\left(\cos b m_{\mathrm{n}} \cosh b m_{\mathrm{n}}+1\right)\left(\sin a m_{\mathrm{n}} \cosh a m_{\mathrm{n}}-\cos a m_{\mathrm{n}} \sinh a m_{\mathrm{n}}\right)\right\}$

$=0$ (Bending),

where $\mu$ is the ratio of the concentrated mass to the mass of the bar and is written as follows:

$\mu=\frac{M}{\rho A l}$.

The suffix $\mathrm{n}$ is the resonance mode number.

If $\mu=0$, Eqs. (2a) and (2b) become

$\sin m_{\mathrm{n} 0}=0$ (Longitudinal),

$\cos m_{\mathrm{n} 0} \cosh m_{\mathrm{n} 0}-1=0$ (Bending),

where the suffix 0 represents the value without the concentrated mass.

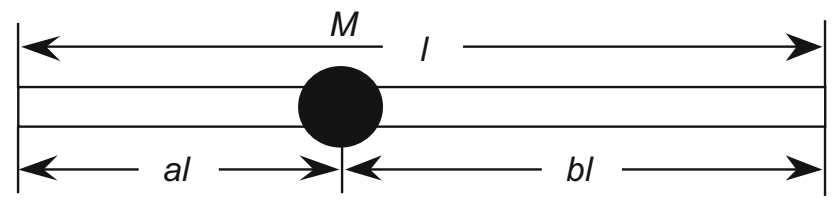

$$
a+b=1,0 \leq a, b \leq 1
$$

Fig. 1 Beam with additional mass
For a bar without a concentrated mass, Eqs. (4a) and (4b) give

$$
\begin{aligned}
m_{\mathrm{n} 0} & =\mathrm{n} \pi(\text { Longitudinal }), \\
m_{10} & =4.730, m_{20}=7.853, m_{30}=10.996, \\
m_{\mathrm{n} 0} & =\frac{1}{2}(2 \mathrm{n}+1) \pi(\mathrm{n}>3) \text { (Bending). }
\end{aligned}
$$

Since the density and Young's modulus are the same before and after the concentrated mass is bound to a specimen, using Eqs. (1a), (1b), (5a), and (5b) results in

$m_{\mathrm{n}}=\frac{f_{\mathrm{n}}}{f_{\mathrm{n} 0}} m_{\mathrm{n} 0}=\frac{f_{\mathrm{n}}}{f_{\mathrm{n} 0}} \mathrm{n} \pi$ (Longitudinal),

$m_{\mathrm{n}}=\sqrt{\frac{f_{\mathrm{n}}}{f_{\mathrm{n} 0}}} m_{\mathrm{n} 0}($ Bending $)$.

The value of $\mu$ can be calculated by substituting $m_{n}$ from Eqs. (6a) and (6b) into Eqs. (2a) and (2b), respectively. By substituting the calculated $\mu$, the concentrated mass, and the dimensions of a bar into Eq. (3), the weight and density can be obtained. The Young's modulus can be calculated by substituting the density from Eq. (3) and the resonance frequency without the concentrated mass into Eqs. (1a) and (1b) [1-13]. This procedure is referred to as the "vibration method with additional mass".

\section{Materials and methods}

\section{Specimens}

Sitka spruce (Picea sitchensis Carr.) was used as the sample specimen in this study. Air-dried specimens $300 \mathrm{~mm}$ long (L, longitudinal), $30 \mathrm{~mm}$ wide (R, radial), and $5 \mathrm{~mm}$ thick ( $\mathrm{T}$, tangential) were placed under water and then conditioned at $20{ }^{\circ} \mathrm{C}$ and $65 \%$ relative humidity (R. H.) till they attained a constant weight. Nine specimens were used and divided into 3 groups of $\mathrm{A}, \mathrm{B}$, and $\mathrm{C}$.

For 3 specimens of Group A, the tests were performed directly after taking the specimen from water $(0 \mathrm{~h})$ and again after 3,6 , and $24 \mathrm{~h}$, and when the weight of the specimen became constant at $20^{\circ} \mathrm{C}$ and $65 \% \mathrm{R}$. H. (5-12 days). When the weight of the specimen became constant at $20^{\circ} \mathrm{C}$ and $65 \%$ R. H., the condition of the specimen is regarded to be air-dried. For 3 specimens of Group B, the tests were performed at 0,9 , and $12 \mathrm{~h}$, and the air-dried condition. For 3 specimens of Group C, the tests were performed at $0,15,18$, and $21 \mathrm{~h}$, and the air-dried condition. After finishing the test under the air-dried condition, the specimens were oven-dried at $105{ }^{\circ} \mathrm{C}$. The oven-dried weight was used to calculate the moisture content of the specimen. Because repeated driving of the staple described later increases the number of holes on the 
RT plane of a specimen, the same specimen was not used from $0 \mathrm{~h}$ to the air-dried condition. The moisture content at $0 \mathrm{~h}$ varied from 93 to $134 \%$.

The following 13 steps shown in Table 1 were applied to the specimens. All tests were conducted at $20{ }^{\circ} \mathrm{C}$ and $65 \%$ R. H.

Step 1: Dimensions and weight of the specimen were measured.

Step 2: The first longitudinal vibration test without the concentrated mass was performed.

Step 3: The specimen was weighed.

Step 4: Longitudinal vibration test with the concentrated mass was performed.

Step 5: The concentrated mass and the specimen were weighed.

Step 6: The second longitudinal vibration test without the concentrated mass was performed.

Step 7: The specimen was weighed.

Step 8: The first bending vibration test without the concentrated mass was performed.

Step 9: The concentrated mass and the specimen were weighed.

Step 10: Bending vibration test with the concentrated mass was performed.

Step 11: The specimen was weighed.

Step 12: The second bending vibration test without the concentrated mass was performed.

Step 13: The specimen was weighed.

The resonance frequency will increase with the decrease in the moisture content caused by drying during vibration tests and the increase in the resonance frequency will affect the accuracy of the vibration method with additional mass.
Hence, the first and second vibration tests without the concentrated mass (steps 2 and 6 for the longitudinal vibration, and Steps 8 and 12 for the bending vibration) were conducted.

\section{Longitudinal vibration test}

To obtain the Young's modulus, free-free longitudinal vibration tests were conducted on the specimen with and without the concentrated mass using the following procedure. The specimen was placed on a small sponge at the position of $x=l / 2$. The longitudinal vibration was generated by tapping one RT plane of the specimen with a wooden hammer $(0.87 \mathrm{~g})$. The motion of the first mode of the bar was detected by a microphone at the other RT plane. The signal was processed through a fast Fourier transform (FFT) digital signal analyzer to yield high-resolution resonance frequencies. A diagram of the experimental setup is shown in Fig. 2.

A normal staple for a stapler shown in Fig. 3 (MAX CO., LTD. MAX STAPLES NO.3-3 M, 0.057 g) was used as the concentrated mass and was driven into the specimen at $x=0$ on the RT plane. Two staples were used for 0-24 $\mathrm{h}$ and one staple was used under the air-dried condition. The specimen weight is smaller at the air-dried condition than the initial condition. The accuracy of the vibration method with additional mass is low for large $\mu$ $[10,14]$. Hence, one staple was used for the air-dried condition, so that $\mu$ is not too large. The values of $\mu$ were 0.00195-0.00430, which were calculated using the masses of the staple and the specimen.

Table 1 Steps of vibration tests

\begin{tabular}{|c|c|c|c|c|c|c|c|}
\hline \multirow[t]{3}{*}{ Step } & \multirow[t]{3}{*}{ Dimension } & \multicolumn{2}{|l|}{ Weight } & \multicolumn{4}{|c|}{ Vibration test } \\
\hline & & \multirow[t]{2}{*}{ Specimen } & \multirow[t]{2}{*}{$M$} & \multicolumn{2}{|c|}{ Longitudinal } & \multicolumn{2}{|l|}{ Bending } \\
\hline & & & & Without $M$ & With $M$ & Without $M$ & With $M$ \\
\hline 1 & 0 & 0 & & & & & \\
\hline 2 & & & & $\bigcirc$ & & & \\
\hline 3 & & $\bigcirc$ & & & & & \\
\hline 4 & & & & & $\bigcirc$ & & \\
\hline 5 & & 0 & $\bigcirc$ & & & & \\
\hline 6 & & & & $\bigcirc$ & & & \\
\hline 7 & & $\bigcirc$ & & & & & \\
\hline 8 & & & & & & $\bigcirc$ & \\
\hline 9 & & O & $\bigcirc$ & & & & \\
\hline 10 & & & & & & & $\bigcirc$ \\
\hline 11 & & O & & & & & \\
\hline 12 & & & & & & ○ & \\
\hline 13 & & 0 & & & & & \\
\hline
\end{tabular}

$M$ concentrated mass 


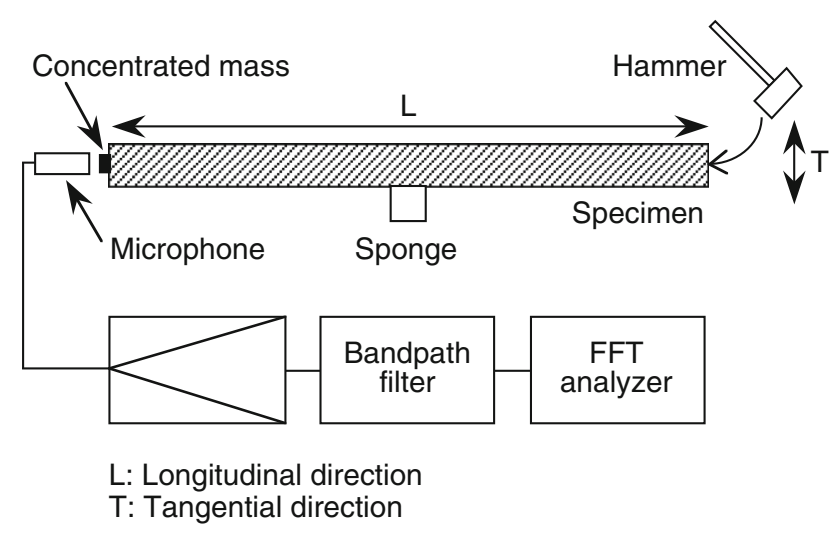

Fig. 2 Schematic diagram of the experimental setup for the free-free longitudinal vibration test

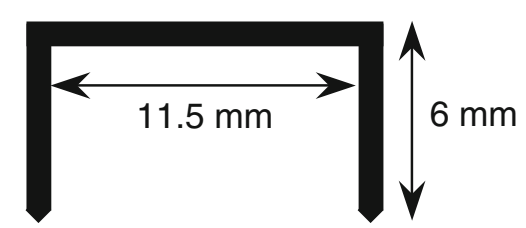

Fig. 3 Staple used for the longitudinal vibration test

\section{Bending vibration test}

To obtain the Young's modulus by bending, free-free bending vibration tests were conducted on the specimen with and without the concentrated mass using the following procedure. The test bar was suspended by two threads at the nodal positions of free-free vibration corresponding to its first resonance mode, and then, the bending vibration was generated by tapping the LR plane of the bar at one end using the same wooden hammer used for the longitudinal vibration test, while bar motion was detected by a microphone at the other end. The signal was processed through the FFT digital signal analyzer to yield high-resolution resonance frequencies (Fig. 4).

An iron plate with dimensions of $2 \mathrm{~mm} \times 3 \mathrm{~mm} \times$ $25 \mathrm{~mm}(1.29 \mathrm{~g})$ was used as the concentrated mass and was bonded at $x=0$ on the LR plane of the specimen with twosided adhesive tape. The values of $\mu$ were $0.0272-0.0649$, which were calculated using the masses of the staple and the specimen.

\section{Results and discussion}

The means (standard deviations) of the density obtained using weight and volume of the specimen and the Young's modulus using free-free longitudinal vibration without the concentrated mass were $521(47.4) \mathrm{kg} / \mathrm{m}^{3}$ and $17.0(2.32)$ $\mathrm{GPa}$, respectively, when the specimen weight became constant.

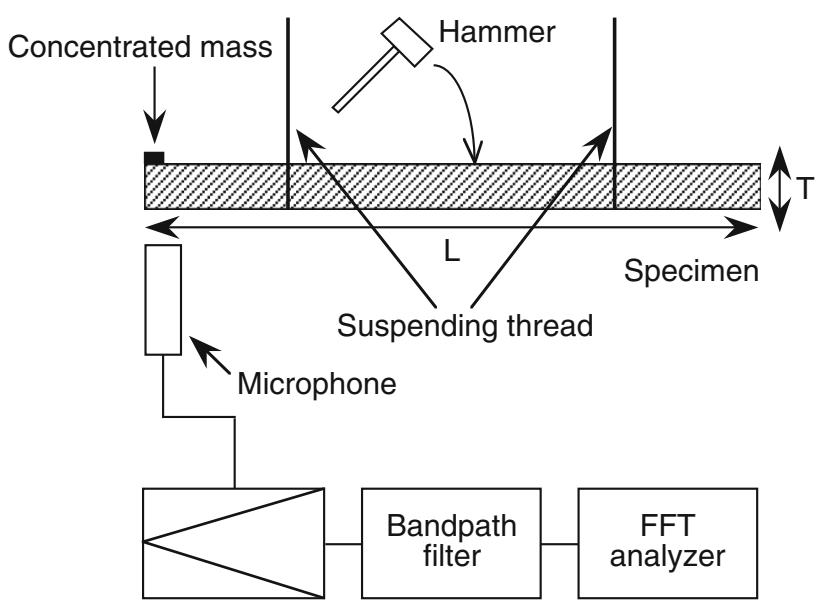

L: Longitudinal direction

$\mathrm{T}$ : Tangential direction

Fig. 4 Schematic diagram of the experimental setup for the free-free bending vibration test

The ratios of weight, density, and Young's modulus, estimated by the vibration method with additional mass, to those obtained by the normal method without the concentrated mass $\left(W / W_{0}, \rho / \rho_{0}, E / E_{0}\right)$ were examined. The weight, density, and Young's modulus by the normal method $W_{0}, \rho_{0}$, and $E_{0}$ were obtained as follows. Weight $W_{0}$ was actual measurement. Density $\rho_{0}$ was calculated from $W_{0}$ and volume of the specimen. Young's modulus $E_{0}$ was calculated using Eqs. (1a), (1b), (5a), and (5b). Resonance frequency was measured without the concentrated mass. These three ratios all show the same values $(W /$ $\left.W_{0}=\rho / \rho_{0}=E / E_{0}\right)$.

Figure 5 shows all results of the changes in the ratios of $W / W_{0}, \rho / \rho_{0}$, and $E / E_{0}$ during drying at $20{ }^{\circ} \mathrm{C}$ and $65 \% \mathrm{R}$. $\mathrm{H}$. When the resonance frequencies without the concentrated mass were values obtained from the first longitudinal and bending vibrations (steps 2 and 8 ), the ratio approached 1 as the moisture content decreased. On the other hand, the ratios were around 1 throughout the drying process when the resonance frequencies without the concentrated mass were values obtained from the second longitudinal and bending vibrations (steps 6 and 12). Therefore, the resonance frequency without the concentrated mass should be measured after measuring the resonance frequency with the concentrated mass.

These trends were caused by the increase in the resonance frequency during the steps $1-13$ as a result of the drying process. Equations (2a) and (2b) are as follows for $a=0$ :

$\mu m_{\mathrm{n}}=-\tan m_{\mathrm{n}}$ (Longitudinal),

$\mu m_{\mathrm{n}}=\frac{1-\cos m_{\mathrm{n}} \cosh m_{\mathrm{n}}}{\cos m_{\mathrm{n}} \sinh m_{\mathrm{n}}-\sin m_{\mathrm{n}} \cosh m_{\mathrm{n}}}$ (Bending). 
Fig. 5 Ratios of weight, density, and Young's modulus estimated by the vibration method with additional mass to those obtained by the normal method without the concentrated mass during drying at $20{ }^{\circ} \mathrm{C}$ and $65 \% \mathrm{R}$. H.
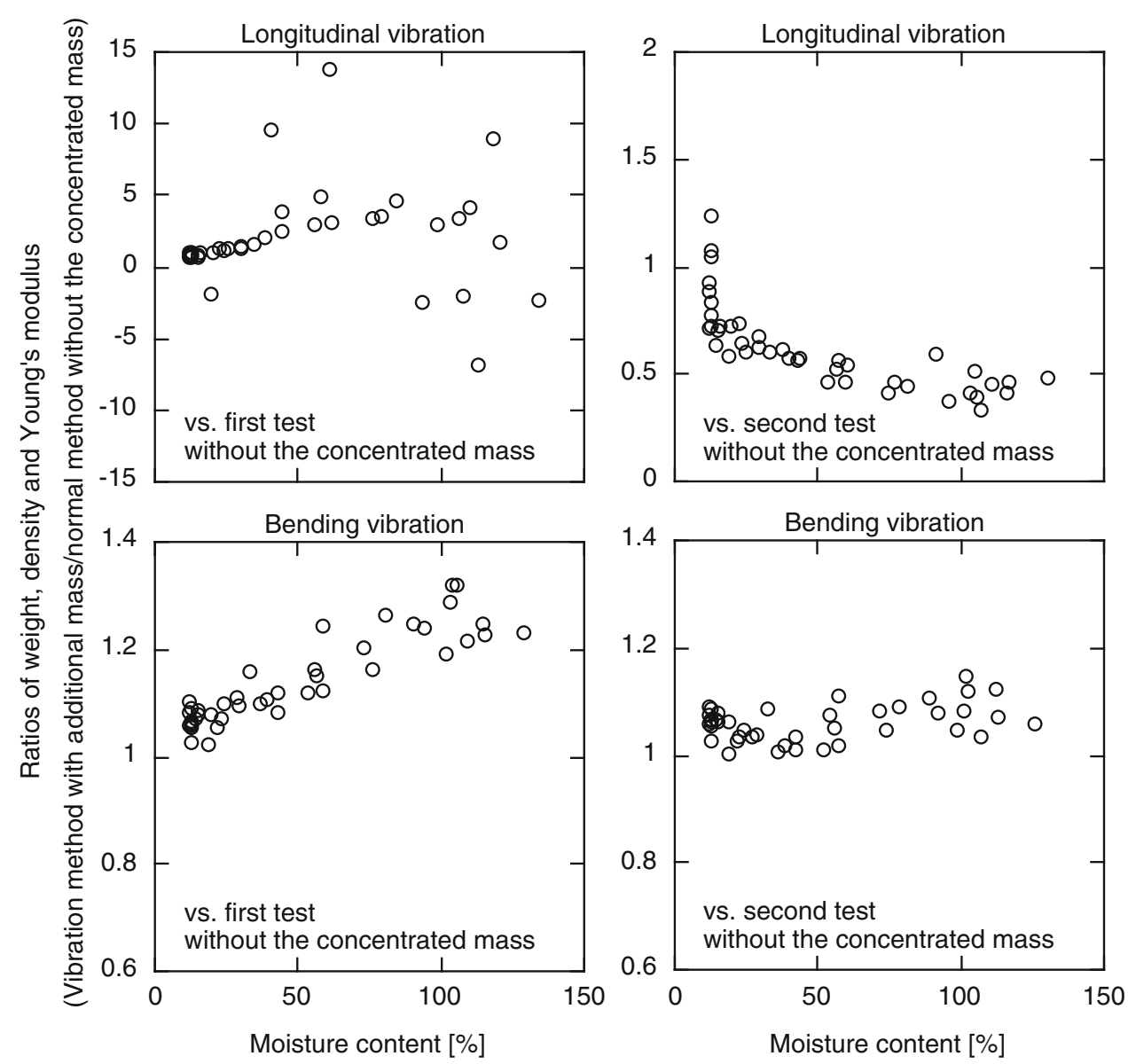

Here, the resonance frequency of the first mode is considered. When the resonance frequency with the concentrated mass $f_{1}$ approaches the resonance frequency without the concentrated mass $f_{10}$, with $f_{1}<f_{10}, m_{1}$ approaches $\pi$ [Longitudinal vibration, Eq. (6a)] and 4.73 [Bending vibration, Eq. (6b)]. For these cases, $\mu$ approaches 0 , and the weight of the specimen from Eq. (3) is very large, as shown in Fig. 6. When $f_{1}>f_{10}, m_{1}$ is larger than $\pi$ (Longitudinal) and 4.73 (Bending). For these cases, $\mu<0$ and the weight of the specimen, from Eq. (3), is less than 0, shown in Fig. 6. Therefore, the accuracy of the vibration method with additional mass was high when the resonance frequencies without the concentrated mass were values from the second longitudinal and bending vibrations (steps 6 and 12).

The ratios for the bending vibration test were nearer 1 than those for the longitudinal vibration test (Fig. 5). This result can be explained by the error in measuring resonance frequencies with and without the concentrated mass, as hereafter. From Eqs. (6a) to (6b),

$y=\pi x$ (Longitudinal), $y=4.73 \sqrt{x}$ (Bending),

where $x=f_{1} / f_{10}, y=m_{1}$. Consequently, the following equations are obtained.

$\frac{\mathrm{d} y}{\mathrm{~d} x}=\pi$ (Longitudinal),

$\frac{\mathrm{d} y}{\mathrm{~d} x}=\frac{2.365}{\sqrt{x}}$ (Bending).

The mean (standard deviation) of $f_{1} / f_{10}$ was 0.936 (0.0133) for steps 8,10 , and 12 . Substituting this value into Eq. (9b) gives $\mathrm{d} y / \mathrm{d} x=2.44$ for bending. Thus, $\mathrm{d} y /$ $\mathrm{d} x$ of the longitudinal vibration is by $29 \%$ greater than that of the bending vibration, which means that the error in $m_{1}$ of the bending vibration is smaller than that of the longitudinal vibration. Therefore, the results of the bending vibration test are more accurate than those of the longitudinal vibration test. However, applying the bending vibration test to each piled lumber is difficult, and this issue should be explored further in the future. 
Fig. 6 Effect of resonance frequency increase caused by drying on the ratio of the concentrated mass to the mass of the specimen during vibration tests
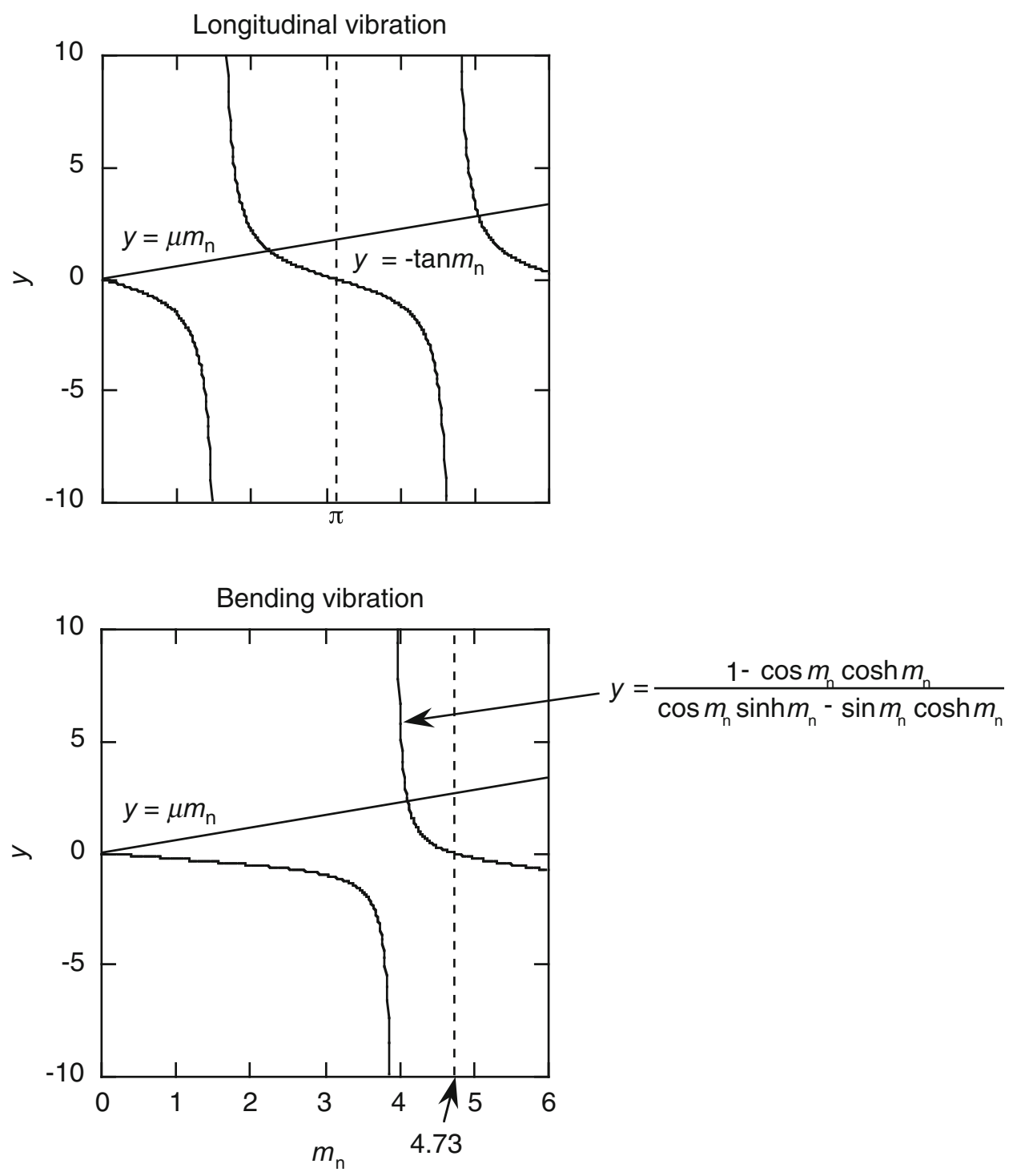

\section{Conclusions}

The resonance frequencies with and without the concentrated mass were measured by longitudinal vibration and bending vibration tests while drying specimens in conditions of $20{ }^{\circ} \mathrm{C}$ and $65 \%$ R. H. and weight, density, and Young's modulus were estimated. The following results were obtained:

1. The accuracy of the estimation was affected by the increase in the resonance frequency during the vibration tests because of drying.

2. The resonance frequency without the concentrated mass should be measured after the measurement of the resonance frequency with the concentrated mass.

3. The accuracy of the bending vibration test estimation was higher than that of the longitudinal vibration test. This was explained by the error caused by measuring resonance frequencies with and without the concentrated mass.

Acknowledgements This study was supported by JSPS KAKENHI Grant number JP15K07522.

\section{References}

1. Chai GB, Low KH (1993) On the natural frequencies of beams carrying a concentrated mass. J Sound Vib 160:161-166

2. Low KH, Lim TM, Chai GB (1993) Experimental and analytical investigations of vibration frequencies for center-loaded beams. Comput Struct 48:1157-1162

3. Low KH (1994) An equivalent-center method for quick frequency analysis of beams carrying a concentrated mass. Comput Struct 50:409-419

4. Skrinar M, Umek A (1994) The influence of an added mass to a change of eigenfrequencies. J Mech Eng 11:443-450 
5. Skrinar M (2002) On elastic beams parameter identification using eigenfrequencies changes and the method of added mass. Comput Mater Sci 25:207-217

6. Kubojima Y, Tonosaki M, Yoshihara H (2003) Effect of the additional mass applied at an end of a wooden beam on its flexural vibrational properties. Wood Ind 58:370-372

7. Kubojima Y, Tonosaki M, Yoshihara H (2005) Effect of additional mass on Young's modulus of a wooden beam. J Test Eval 33:278-282

8. Türker T, Bayraktar A (2008) Structural parameter identification of fixed end beams by inverse method using measured natural frequencies. Shock Vib 15:505-515

9. Kubojima Y, Suzuki Y, Tonosaki M (2014) Effect of additional mass on the apparent Young's modulus of a wooden bar by longitudinal vibration. BioRes 9:5088-5098

10. Kubojima Y, Sonoda S (2015) Measuring Young's modulus of a wooden bar using longitudinal vibration without measuring its weight. Eur J Wood Wood Prod 73:399-401

11. Matsubara M, Aono A, Kawamura S (2015) Experimental identification of structural properties of elastic beam with homogeneous and uniform cross section. Trans JSME. doi:10. 1299/transjsme.15-00279

12. Kubojima Y, Kato H, Tonosaki M, Sonoda S (2016) Measuring Young's modulus of a wooden bar using flexural vibration without measuring its weight. BioRes 11:800-810

13. Matsubara M, Aono A, Ise T, Kawamura S (2016) Study on identification method of line density of the elastic beam under unknown boundary conditions. Trans JSME. doi:10.1299/trans jsme.15-00669

14. Sonoda S, Kubojima Y, Kato H (2016) Practical techniques for the vibration method with additional mass Part 2: experimental study on the additional mass in longitudinal vibration test for timber measurement. In: CD-ROM Proceedings of the World conference on timber engineering (WCTE 2016)

15. Kubojima Y, Sonoda S, Kato H (2017) Practical techniques for the vibration method with additional mass: effect of crossers' position in longitudinal vibration. J Wood Sci 63:147-153 